\title{
Effect of Modified Hydrotalcites on Flame Retardancy and Physical Properties of Paper
}

\author{
Songlin Wang, ${ }^{\mathrm{a}, \mathrm{b}, *}$ Xuxu Yang, ${ }^{\mathrm{a}}$ Fei Wang, ${ }^{\mathrm{a}}$ Zongjia Song, ${ }^{\mathrm{a}}$ Hehe Dong, ${ }^{\mathrm{a}}$ and Luqing Cui ${ }^{\mathrm{a}}$ \\ Functionalized layered double hydroxides (LDHs) based on a multi- \\ modifier system composed of itaconic acid (ITA) and titanate coupling \\ agent (NDZ-201) were designed and fabricated in this paper with the aim \\ to develop high-performance fire retardant paper. The structure of LDHs \\ were characterized using Fourier transform infrared spectroscopic \\ analysis, X-ray diffraction analysis, thermogravimetric analysis (TGA), \\ scanning electron microscopic analysis, cone calorimetry, and laser \\ particle size analysis. The results showed that carbonate anions were \\ partially replaced by ITA, whereas the titanate coupling agent was \\ attached to the surfaces of the hydrotalcites. The limiting oxygen index \\ (LOI), TGA curves, total heat release rate (THR), and heat release rate \\ (HRR) indicated that as the addition of hydrotalcites increased, the \\ modified LDHs' LOI value and thermal stability noticeably increased \\ compared to the unmodified hydrotalcites, and the HRR and THR of the \\ material decreased. When the addition amount was $25 \%$, the LOI of ITA- \\ LDHs was $26.9 \%$. However, the Mg-Al hydrotalcites adversely affected the \\ strength index of flame-retardant paper; the modified hydrotalcites clearly \\ reduced this effect, and the whiteness of paper increased and reached \\ $83 \%$.
}

Keywords: Modified filler composite; Hydrotalcite; Paper; Flame retardancy; Physical strength properties

Contact information: a: College of Marine Science and Biological Engineering, Shandong Provincial Key Laboratory of Biochemical Engineering, Qingdao University of Science \& Technology, Qingdao, Shandong 266042 China; $b$ : Key Laboratory of Pulp and Paper Science \& Technology of Ministry of Education/Shandong Province, Qilu University of Technology, Jinan, Shandong 250353 China;

* Corresponding author: wangsongl@126.com

\section{INTRODUCTION}

Since the start of the twenty-first century, environmental issues and the depletion of petroleum resources have led to more focused research for the need to replace petroleum and petroleum-based products with natural and renewable resources (Köklükaya et al. 2015; $\mathrm{Si}$ and Guo 2016). The cellulose obtained from wood and cotton is one of the earth's most abundant biopolymers found in nature (Weinstock et al. 1997). Wood-derived cellulose has been the main raw material used in paper manufacturing for over a century. Paper can be described as a three-dimensional fibrous network formed from the filtration of an aqueous pulp fiber slurry; the formed fiber network is biodegradable, recyclable, and inexpensive (Horrocks et al. 2015). It is for these reasons that paper and fibre networks are used in many fields, such as corrugated fiberboard, packaging materials, printed substrates, cleaning, and sanitary products. Although cellulose fibers have many advantages, their high flammability is a major disadvantage, which limits their use in other applications. Several studies have investigated methods to increase the flame retardancy of cotton and cellulose materials. 
In the process of papermaking or as the post-treatment of paper, the paper-based products show flame retardancy after adding flame retardant additives to the fiber wall (Horrocks 1996). The most widely used additives contain inorganics such as phosphorus, halogens, boron, silicates, hydroxides, and hydrotalcites (Nassar et al. 2015).

Hydrotalcites are a type of anionic-layered inorganic, also known as layered double hydroxides (LDHs) (Reichle et al. 1986; Li and Duan 2006). They are composed of laminate metal cations and interlayer anions that are modulated over a certain range (Khan and O'Hare 2002; Liu et al. 2006). These layered inorganics are widely used in the fields of catalysts, adsorbents, heat stabilizers, flame retardants, etc. (Choudary et al. 2002; Pereira et al. 2009; Mahmoud et al. 2017). As opposed to traditional inorganics and halogenated flame retardants, LDHs not only exhibit high flame-retardant efficiency, but are also non-toxic, halogen-free, and suppress smoke formation. These materials are becoming the new generation of environmentally compatible flame retardants (Becker et al. 2011). Hence, LDHs could be used as an inorganic filler material in paper to improve its whiteness and incombustibility (Wang and Song 2010). Hydrotalcites are easily agglomerated and are incompatible with organic substances; hence, these fillers need to be treated with organic modifiers. Commonly used modifiers include organic acids, various surfactants, and coupling agents (Costantino et al. 2009; Wang and O'Hare 2012).

Zhang et al. (2016) used inorganic MgAl-LDH flame retardant to prepare flame retardant medium fiberboard (MDF). The results showed that the fire retardant and smoke suppression effects were more effective when the MgAl-LDH levels increased. When the MgAl-LDH level was $15 \%$, the heat release effect was similar to MDF made with $10 \%$ MP but showed superior smoke prevention. The more MgAl-LDH that was used, the weaker became the mechanical properties of the MDF. Yan et al. (2017) successfully prepared a novel sulfamic acid intercalated MgAl-LDH (SA-LDH) and used to improve the flame retardancy of EVA co-polymer. The results showed that the composite sample containing $19.0 \mathrm{wt} \%$ IFR and $1.0 \mathrm{wt} \%$ SA-LDH achieved the maximal LOI value of $48.7 \%$, passed the UL-94 V-0 rating, and significantly decreased the peak heat release rate from 306.3 $\mathrm{kW} / \mathrm{m}^{2}$ of neat PLA to $58.1 \mathrm{~kW} / \mathrm{m}^{2}$.

Kalali et al. (2015) prepared functionalized LDHs that were based on a multimodifier system, i.e., sodium salt of hydroxypropyl-sulfobutyl- $\beta$-cyclodextrin (sCD) that was combined with dodecylbenzenesulfonate (DBS) and taurine (T). The layer spacing of the modified LDHs was expanded, and its dispersibility in an epoxy resin was improved when compared to the unmodified LDH. The limiting oxygen index (LOI) and char formation rate were improved with the modifications. Additionally, the UL-94 vertical combustion test rated the modified LDHs at the V0 level. The modified LDHs exhibited high flame retardancy under the same packing load.

In this paper, a hydrotalcite was intercalated with itaconic acid (ITA-LDHs) and then its surfaces were covered with a titanate coupling agent (NDZ-201); the intercalated hydrotalcite was synthesized using an anion exchange method. The structure of the modified hydrotalcite was characterized using Fourier transform infrared (FTIR) spectroscopy, X-ray diffraction (XRD) analysis, thermogravimetric analysis (TGA), and scanning electron microscopy (SEM) analysis. Unmodified and modified hydrotalcites were added to the pulp slurry as an inorganic filler, and the resulting handsheets made from the slurries were tested for their flame retardancy and physical strength properties. 


\section{EXPERIMENTAL}

\section{Materials}

Magnesium nitrate hexahydrate $\left(\mathrm{Mg}\left(\mathrm{NO}_{3}\right)_{2} \cdot 6 \mathrm{H}_{2} \mathrm{O}\right)$ and anhydrous sodium carbonate $\left(\mathrm{Na}_{2} \mathrm{CO}_{3}\right)$ were purchased from Tianjin Dingsheng Xin Chemical Co., Ltd. (Tianjin, China). Aluminum nitrate nonahydrate $\left(\mathrm{Al}\left(\mathrm{NO}_{3}\right)_{3} \cdot 9 \mathrm{H}_{2} \mathrm{O}\right)$ was obtained from Tianjin Guangcheng Chemical Reagent Co., Ltd. (Tianjin, China). Sodium hydroxide (flakes) and itaconic acid were purchased from National Drug Group Chemical Reagents Co., Ltd. (Shanghai, China). Anhydrous ethanol was obtained from a chemical reagent company (Tianjin Bohuatong, Tianjin, China). All of the above reagents were of analytical grade. A titanate coupling agent (NDZ-201) was purchased from Nanjing Pinning Coupling Agent Co., Ltd. (Nanjing, China), and was of industrial grade. Softwood pulp was obtained from a pulp mill located in the Shandong Province (China). The pulp's moisture content was $79.58 \%$ (wt), and had a drainage freeness of $37.8{ }^{\circ} \mathrm{SR}$. Deionized water was used in the preparation and washing processes. Polyacrylamide was purchased from National Drug Group Chemical Reagents Co., Ltd. (Shanghai, China); the polymer had a molecular weight of 5 million.

The equipment used consisted of a FTIR spectrometer (VECTOR22; Germany Brooke Co., Ettlingen, Germany), X-ray diffractometer (D/max-2500; Japan Science Co., Shanghai, China), scanning electron microscope (JSM-6380; Japan JEOL Company, Beijing, China), thermogravimetric analyzer (TAQ-500; Shimadzu Co., Kyoto, Japan), laser particle size analyzer (Mastersizer 3000; Malvern Panalytical Co., Shanghai, China), cone calorimeter (FTT0030; FTT Co., London, England), and oxygen index tester (JF-3; Nanjing Jiangning District Analysis Instrument Factory, Nanjing, China).

\section{Preparation of $\mathrm{LDHs}$}

The $\mathrm{Mg}\left(\mathrm{NO}_{3}\right)_{2} \cdot 6 \mathrm{H}_{2} \mathrm{O}$ and $\mathrm{Al}\left(\mathrm{NO}_{3}\right)_{3} \cdot 9 \mathrm{H}_{2} \mathrm{O}$ (at a $n_{\mathrm{Mg}}: n_{\mathrm{Al}}=2: 1$ ratio) were dissolved into $100 \mathrm{~mL}$ of deionized water to form a salt solution. A total of $0.15 \mathrm{~mol} / \mathrm{L}$ of NaOH and $0.05 \mathrm{~mol} / \mathrm{L}$ of $\mathrm{Na}_{2} \mathrm{CO}_{3}$ were dissolved into $100 \mathrm{~mL}$ of deionized water to form an alkaline solution. Then, the salt solution and alkali solution were slowly added to three mouthed flasks at room temperature $\left(25^{\circ} \mathrm{C}\right)$. During the addition, the $\mathrm{pH}$ of the solution was kept around 10 to 11 . After the addition was completed, the mixture was stirred at room temperature for $1 \mathrm{~h}$; then, the temperature was raised to $65^{\circ} \mathrm{C}$ and maintained for 18 to 20 $\mathrm{h}$. The resulting slurry was cooled, filtered, and washed two to three times with deionized water. The washed filter cake was dried at $80{ }^{\circ} \mathrm{C}$.

\section{Preparation of ITA-LDHs}

The $\mathrm{Mg}\left(\mathrm{NO}_{3}\right)_{2} \cdot 6 \mathrm{H}_{2} \mathrm{O}$ and $\mathrm{Al}\left(\mathrm{NO}_{3}\right)_{3} \cdot 9 \mathrm{H}_{2} \mathrm{O}$ (at a $n_{\mathrm{Mg}}: n_{\mathrm{Al}}=2: 1$ ratio) were dissolved into $100 \mathrm{~mL}$ of deionized water to form a salt solution. A total of $0.15 \mathrm{~mol} / \mathrm{L}$ of $\mathrm{NaOH}$ and $0.05 \mathrm{~mol} / \mathrm{L}$ of $\mathrm{Na}_{2} \mathrm{CO}_{3}$ were dissolved into $100 \mathrm{~mL}$ of deionized water to form an alkaline solution. Then, the salt solution and alkali solution were slowly added to three mouthed flasks at room temperature $\left(25^{\circ} \mathrm{C}\right)$. During the addition, the $\mathrm{pH}$ of the solution was kept around 10 to 11 . After completion of the addition, the solution was stirred for $0.5 \mathrm{~h}$, heated to $65^{\circ} \mathrm{C}$; then, $2.5 \mathrm{~g}$ of itaconic acid were dissolved in $50 \mathrm{~mL}$ of water and pour them into three flasks, stirred at constant temperature for $1 \mathrm{~h}$, and then $2.5 \mathrm{~g}$ titanate coupling agent (NDZ-201) was added, and the reaction solution was stirred for $2 \mathrm{~h}$. Then stirring was stopped and the mixture was held for $18 \mathrm{~h}$ at this temperature. The slurry obtained after cooling was filtered and washed two to three times with deionized water. The washed filter 
cake was dried at $80{ }^{\circ} \mathrm{C}$.

\section{Preparation of flame-retardant paper handsheets}

The wet-lap softwood pulp from the mill was disintegrated in a standard disintegrator at 30,000 revolutions at $1 \%$ consistency in water; the LDHs were then added to the disintegrated slurry at 0 to $25 \%$ on a dry pulp mass basis. The resulting slurry was diluted to $0.5 \%$ consistency with deionized water. The dosage of cationic polyacrylamide used was $0.03 \%$, and its intended function was as a retention aid. Laboratory paper handsheets were made from the prepared pulp slurry on a sheet former at a grammage of $80 \mathrm{~g} / \mathrm{m}^{2}$.

\section{Methods}

FTIR analysis of LDHs and ITA-LDHs

Spectra were recorded in a wavenumber range from 500 to $4000 \mathrm{~cm}^{-1}$. The sample powder and $\mathrm{KBr}$ were uniformly mixed, and then compressed into disks to be analyzed by FTIR.

$X R D$ analysis of $L D H s$ and ITA-LDHs

The X-ray diffractometer used $\mathrm{Cu} \mathrm{K} \alpha$ radiation $(\lambda=0.154 \mathrm{~nm}$ wavelength) at a scanning speed of $3^{\circ} \% \mathrm{~min}$ over the scanning range of $5^{\circ}$ to $80^{\circ}$.

\section{TGA of LDHs and ITA-LDHs}

The test sample ( $c$. $10 \mathrm{mg}$ ) was placed into a nitrogen atmosphere, and the temperature was increased from room temperature to $700{ }^{\circ} \mathrm{C}$ at a rate of $10{ }^{\circ} \mathrm{C} / \mathrm{min}$. The nitrogen flow rate during the TGA measurements was controlled at $50 \mathrm{~mL} / \mathrm{min}$.

\section{SEM analysis of LDHs and ITA-LDHs}

The SEM micrographs (JSM-6380; Japan JEOL Company, Beijing, China) of the nanoparticles were recorded to characterize the structures of LDHs before and after the modification. The samples were dispersed in methanol and dripped on silicon wafer. All of the samples were sputter-coated in gold prior to the observation (Shensei Scientific Instruments Co., Ltd., Shenzhen, China).

Particle size analyzer (PSA)

A small amount of LDHs were diluted in distilled water at a ratio of 1:10. The particle size distribution of the LDHs were measured using the Malvern ZetaSizer 3000 laser particle size analyzer (Shanghai, China).

\section{Limiting oxygen index of flame-retardant paper}

To test the flame retardancy of paper, the limiting oxygen index of flame-retardant paper was measured using a JF-3 oxygen index instrument (Jiangning analytical instrument Co., Ltd., Nanjing, China) and according to the GB/T 14656 (2009) standard.

\section{Cone calorimeter analyzer}

The thermal radiation power was $35 \mathrm{~kW} / \mathrm{m}^{2}$. The mass of all samples were $10 \mathrm{~g}$, the addition of hydrotalcites were $25 \%$, and the size was $10 \mathrm{~mm} \times 10 \mathrm{~mm} \times 5 \mathrm{~mm}$. The total heat release rate (THR) and heat release rate (HRR) of the samples were measured. 
Detection of the physical properties of the flame-retardant paper

The tensile index, burst index, and whiteness were measured according to GB/T 12914 (2008), GB/T 454 (2002), and QB/T 2804 (2006), respectively.

\section{RESULTS AND DISCUSSION}

\section{Analysis of Infrared Spectrum of LDHs and ITA-LDHs}

Figure 1 shows the infrared spectra of the LDHs and ITA-LDHs. The broad absorption peak at $3461 \mathrm{~cm}^{-1}$ was attributed to the $-\mathrm{OH}$ vibration of the physically adsorbed water or the hydrated crystal water, or to the stretching vibration of the $\mathrm{M}-\mathrm{OH}$ bond (where " $\mathrm{M}$ " is $\mathrm{Al}$ or $\mathrm{Mg}$ ). The absorption peak at $1353 \mathrm{~cm}^{-1}$ was attributed to the symmetrical stretching vibration of the interlayer carboxylate anion. The peak of $831 \mathrm{~cm}^{-1}$ was ascribed to the $\mathrm{Mg}-\mathrm{O}-\mathrm{Al}$ stretching vibration of the $\mathrm{LDHs}$ laminate. The absorption peak located at $661 \mathrm{~cm}^{-1}$ was attributed to the bending vibration of $\mathrm{Mg}-\mathrm{OH}-\mathrm{Al}$.

When compared to the unmodified LDHs, the absorption peaks at $2875 \mathrm{~cm}^{-1}, 2960$ $\mathrm{cm}^{-1}$, and $2932 \mathrm{~cm}^{-1}$ in the modified LDHS are characteristic of antisymmetric vibrations of methyl and methylene groups. A vibrational absorption at $1382 \mathrm{~cm}^{-1}$ indicated that there were some carboxylate anions present in the modified hydrotalcite structure. The asymmetric and symmetric stretching absorptions of the carboxylate anion at $1641 \mathrm{~cm}^{-1}$ and $1465 \mathrm{~cm}^{-1}$, respectively, were observed. There was observed evidence of phosphate present in the modified LDHs. The FTIR showed $\mathrm{P}=\mathrm{O}$ stretching vibration at $1207 \mathrm{~cm}^{-1}$ and $\mathrm{P}-\mathrm{O}$ stretching vibration at $1033 \mathrm{~cm}^{-1}$. An out-of-plane rocking absorption peak of a $=\mathrm{CH}_{2}$ group was noted at $825 \mathrm{~cm}^{-1}$. These observed absorption peaks suggested that itaconic acid and titanate coupling agent (i.e., NDZ-201) are presented in the modified LDH structures.

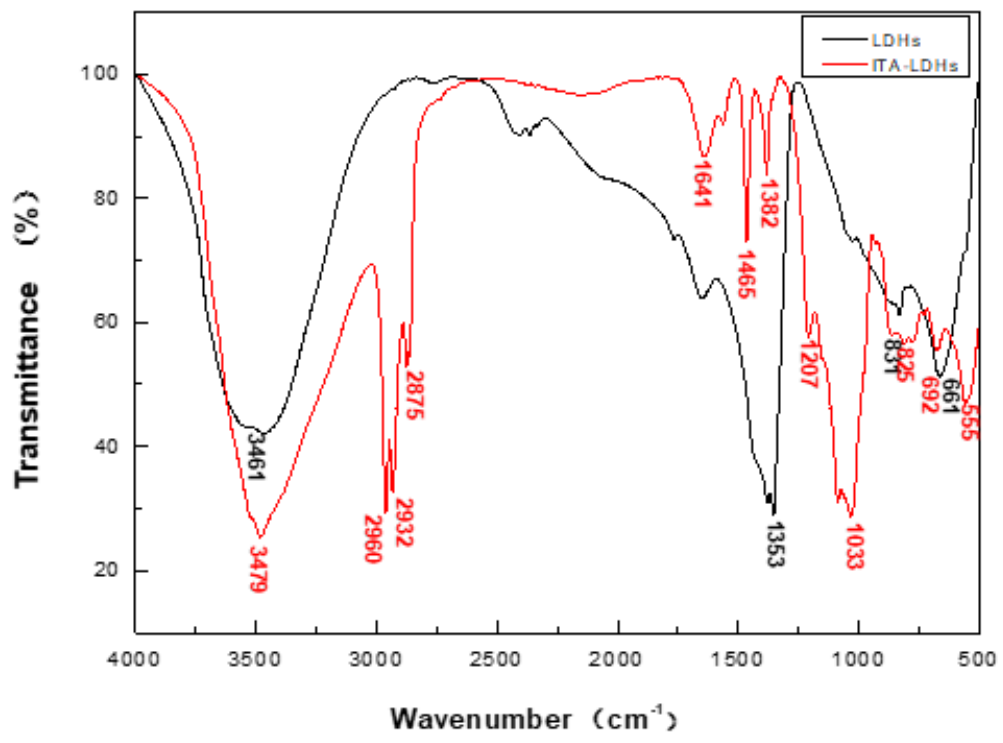

Fig. 1. Infrared spectrum of LDHs and ITA-LDHs

\section{Analysis of X-ray Diffraction of LDHs and ITA-LDHs}

Figure 2 shows the diffraction peaks of the (003), (006), (009), and (110) planes with relatively high diffraction intensity for the LDHs examined. According to Bragg's 
Law (i.e., $2 d \bullet(\sin \theta)=n \lambda)$, the interlayer spacing $(d)$ calculated from the diffraction peaks from the (003) plane in the unmodified LDH was $0.80 \mathrm{~nm}$. For the (006) plane, the $d$ value was calculated as $0.42 \mathrm{~nm}$. The $d$ value for the (009) plane was calculated as $0.26 \mathrm{~nm}$, whereas for the (110) plane, the $d$ value was calculated as $0.15 \mathrm{~nm}$. In the ITA-LDHs, the diffractions for the (009) and (110) planes disappeared, whereas the (006) plane diffraction intensity weakened. In contrast, the diffraction intensity of the (003) plane became stronger. The intercalation ratio $(a)$ of the modified hydrotalcite was calculated using Eq. 1 (Gardolinski et al. 2000),

$$
a(\%)=\left(\frac{I i(003)}{\operatorname{Ii(003)}+\operatorname{Ik}(003)}\right) \times 100
$$

where $I_{\mathrm{i}}(003)$ is the diffraction intensity of the (003) plane after being intercalated and $I_{\mathrm{k}(003)}$ is the diffraction intensity of the unmodified hydrotalcite $(003)$ plane .

After the modification, an intercalation ratio of $69.7 \%$ was obtained with the modified LDHs. The intercalation was not entirely due to the lower adsorption degree of monovalent ions in the aqueous solution versus the polyvalent ions. Secondly, due to the presence of a small amount of carbonate anions, it was very difficult to completely remove them during the preparation and the synthesis of the hydrotalcite, as well as during the washing, filtering, and drying steps after the synthesis. The spacing of the (003) planes increased from $0.80 \mathrm{~nm}$ to $2.42 \mathrm{~nm}$, indicating that larger organic anions entered the LDHs layer, which increased the interlamellar spacing. According to Shi et al. (2010), the use of itaconic acid alone to modify hydrotalcite can increase the interlayer spacing of the (003) planes to $0.98 \mathrm{~nm}$. This reported value is less than the $2.42 \mathrm{~nm}$ plane spacing observed in this study.

These observations indicated that not only itaconic acid, but also some titanate coupling agents were intercalated into the LDHs interlayer. From these X-ray diffraction analyses combined with the earlier FTIR analyses, it was concluded that itaconic acid and titanate coupling agents had been incorporated into the modified LDHs.

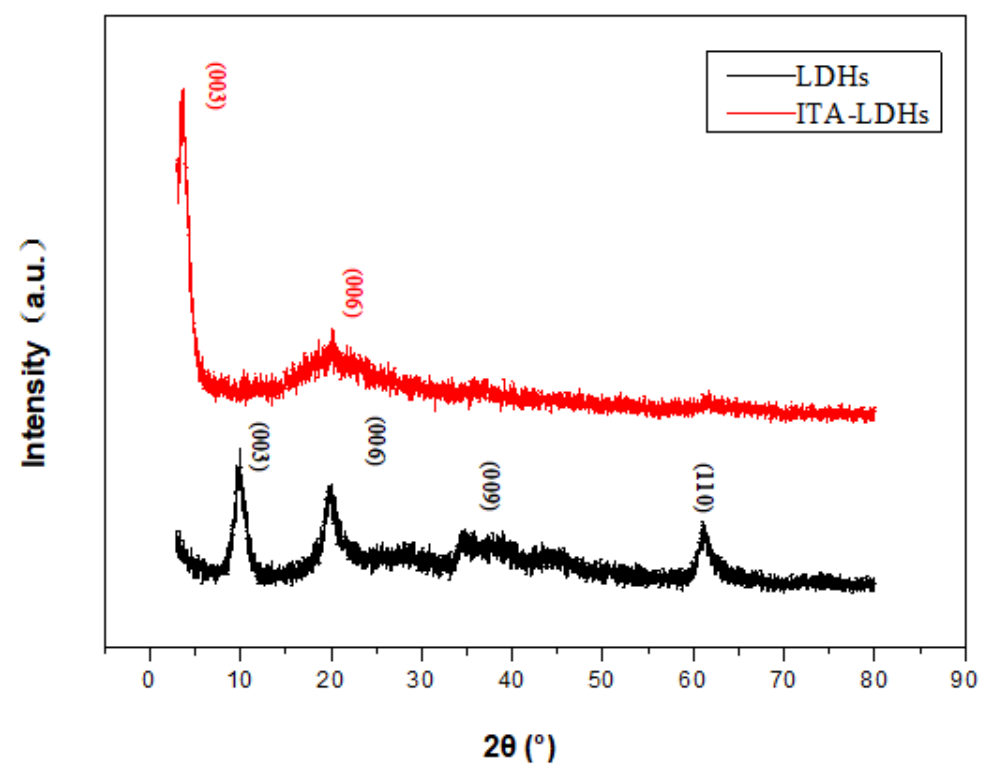

Fig. 2. X-ray diffraction patterns of the LDHs and ITA-LDHs 


\section{Thermogravimetric Analysis of LDHs}

Figure 3 shows the recorded thermogravimetric curves for the unmodified hydrotalcite when it was heated from $17{ }^{\circ} \mathrm{C}$ to $700{ }^{\circ} \mathrm{C}$ at $10^{\circ} \mathrm{C} / \mathrm{min}$. The mass loss of the hydrotalcite can be described as follows. The first stage occurred between 17 to $233{ }^{\circ} \mathrm{C}$ and the mass loss rate was approximately $11.38 \%$. The first stage was mainly attributed to the removal of physically-adsorbed and interlayered water; in this stage, the interlayer structure of LDHs was not degraded. In the second stage, which occurred over 233 to 365 ${ }^{\circ} \mathrm{C}$, the mass loss rate was approximately $6.66 \%$. The mass loss in the second stage was mainly due to the decomposition of the lamina $-\mathrm{OH}$ groups, where the layered structure of the LDHs was gradually degraded. In the third stage, which occurred at 365 to $700{ }^{\circ} \mathrm{C}$, the mass loss rate was approximately $30.85 \%$. This mass loss was mainly attributed to the dehydroxylation of the LDHs laminates, the formation of magnesium aluminum oxides, and the evolution of carbon dioxide gas from the decomposition of the carbonate interlayer.

The mass loss rates of the ITA-LDHs in the first, second, and third stages were $7.5 \%, 46.4 \%$, and $10.8 \%$, respectively. The mass loss rate of the modified LDHs in the first stage was appreciably lower than that of the unmodified LDHs. This was attributed to the modified LDHs being more hydrophobic (i.e., more lipophilic), which was caused by the lower water adsorption on the surfaces and the slower water removal rate between the layers. When the temperature was increased above $188{ }^{\circ} \mathrm{C}$, the itaconic acid began to decompose, which resulted in the accelerated thermal decomposition of the modified LDHs; the mass loss rate at this stage was considerably higher than that of the unmodified LDHs.

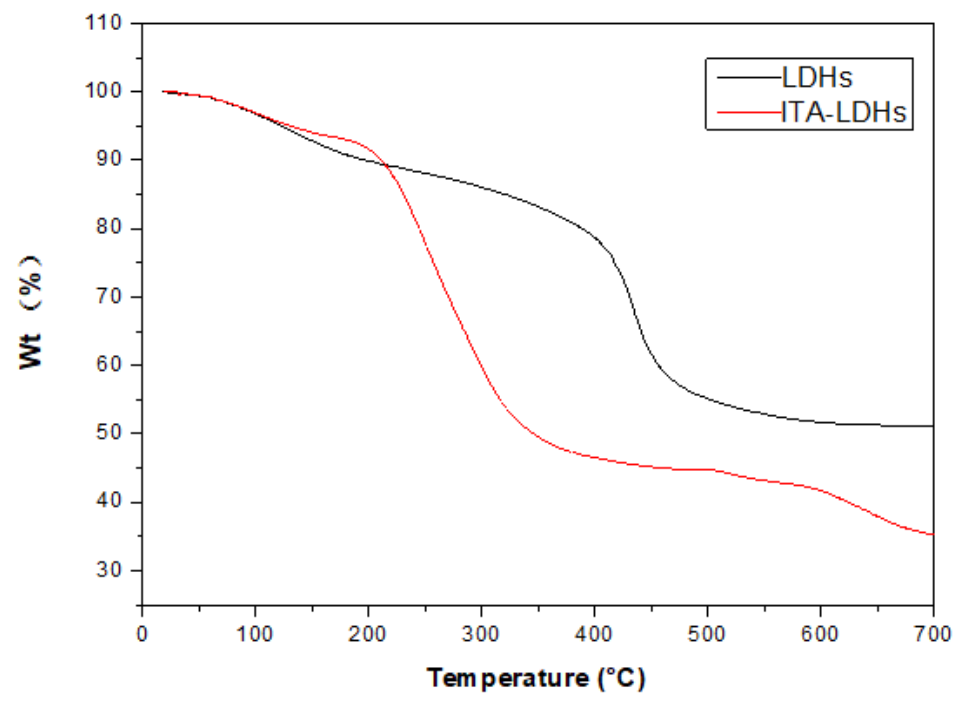

Fig. 3. Recorded TGA curves of LDHs and ITA-LDHs

\section{SEM and Particle Size Analysis}

In Fig. 4a, the unmodified LDHs particles were appreciably aggregated to one another. This was due to the formation of hydrogen bonds on the surface of the unmodified LDHs, resulting in soft agglomeration and formation of large particles with irregular shapes and sizes. In Fig. 4b, the degree of reunion of the modified LDHs was obviously reduced, and the particle size decreased.

Figure 5 indicated that the average particle size of the modified LDHs was $1.5 \mu \mathrm{m}$, whereas the unmodified LDHs had an average particle size of $2.86 \mu \mathrm{m}$. The particle size 
distribution for the modified LDHs was narrower than that of the unmodified LDHs, which indicated that titanate coupling agent was attached to the surfaces of the hydrotalcite particles. According to Li et al. (2014), it is known that the same addition of a flame retardant results in smaller particle sizes, narrower particle size distribution, better dispersion in the substrate, and a better flame-retardant effect. It was concluded that the flame-retardant effect of the modified LDHs was better than that of the unmodified LDHs.
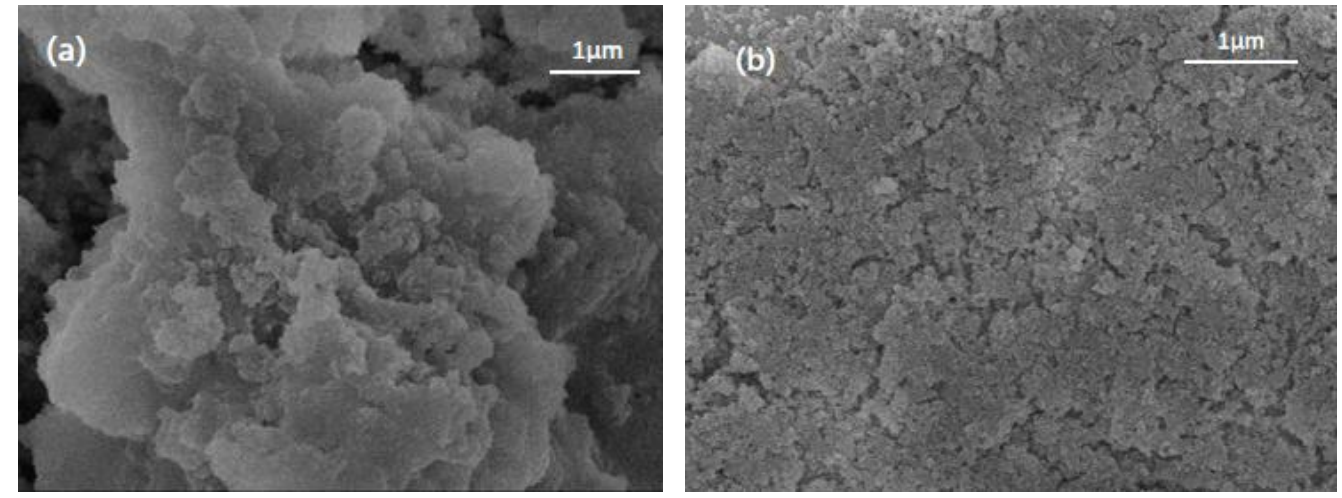

Fig. 4. SEM images of LDHs and ITA-LDHs. a: LDHs; b: ITA-LDHs

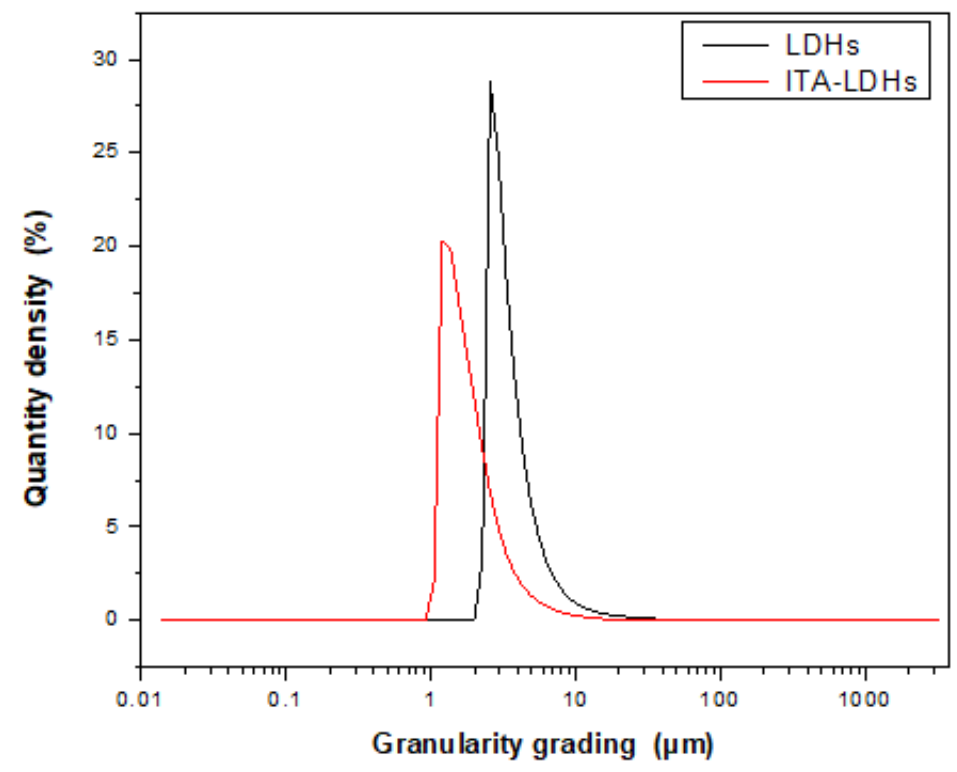

Fig. 5. Particle size distributions of LDHs and ITA-LDHs

\section{Flame Retardancy of Paper Handsheets Made with LDHs}

Figure 6 shows that the LOI value of handsheets gradually increased as the applied dosages of unmodified LDHs and ITA-LDHs increased. Furthermore, it was observed that the LOI of the ITA-LDHs handsheets was higher than that of the LDHs handsheets at the same dosage level. This observation indicated that the LOI of pure handsheets was $17.5 \%$, and the modified hydrotalcites had a higher flame-retardant effect than the unmodified hydrotalcites. When the dosage was $20 \%$ on pulp, the LOI of the ITA-LDHs handsheets was $26.5 \%$, which was much higher than unmodified LDHs handsheets (i.e., 24.1\%). 


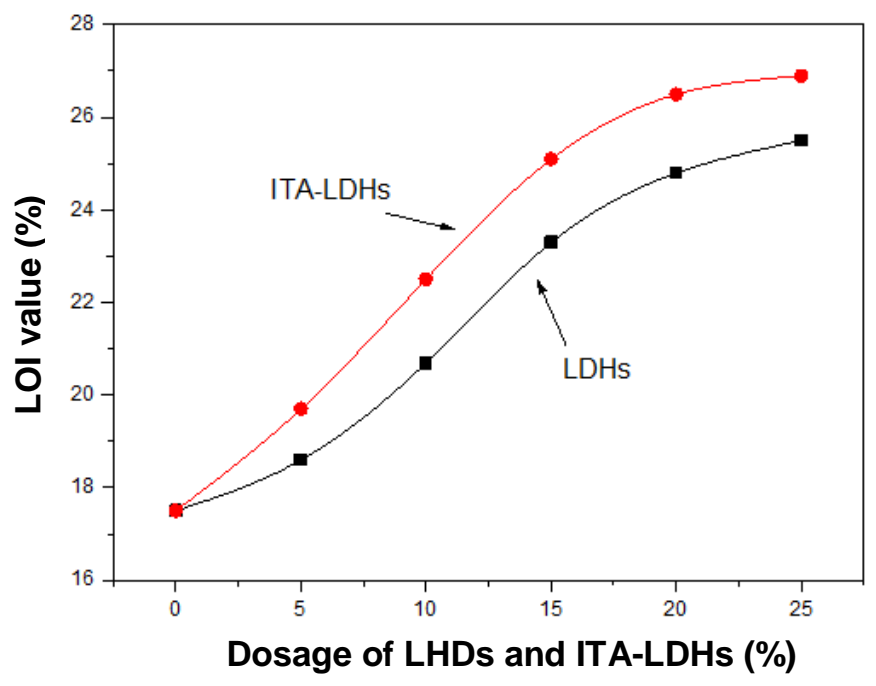

Fig. 6. Effect of dosage of LDHs and ITA-LDHs on paper handsheet LOI values

\section{TGA of Paper Handsheets with LDHs}

The thermal mass loss analysis (Fig. 7) indicated that the addition of unmodified LDHs improved the thermal stability of the formed paper handsheets to a small degree, whereas the addition of modified ITA-LDHs improved the thermal stability to a much higher degree when compared to the control handsheets without LDHs. The unmodified hydrotalcite particles varied in size. It was difficult for the flame-retardant LDHs to bond and to form a uniform composite with the formed paper handsheet; this resulted in uneven distributions of LDHs within the paper matrix of fibers and fillers. The ITA-LDHs handsheets had higher flame retardancy than the LDHs handsheets, which resulted in improved thermal stability of the ITA-LDHs handsheets. It was observed that the handsheets containing either the unmodified or the modified LDHs had lower thermal mass losses at $700{ }^{\circ} \mathrm{C}$ than the control handsheets (without LDHs); the residual rate of pure handsheets at $700{ }^{\circ} \mathrm{C}$ was only $8.9 \%$, and the handsheets that had the unmodified LDHs was 33.5\%; and the handsheets that had the modified LDHs had a lower thermal mass loss of $53.9 \%$ at $700{ }^{\circ} \mathrm{C}$.

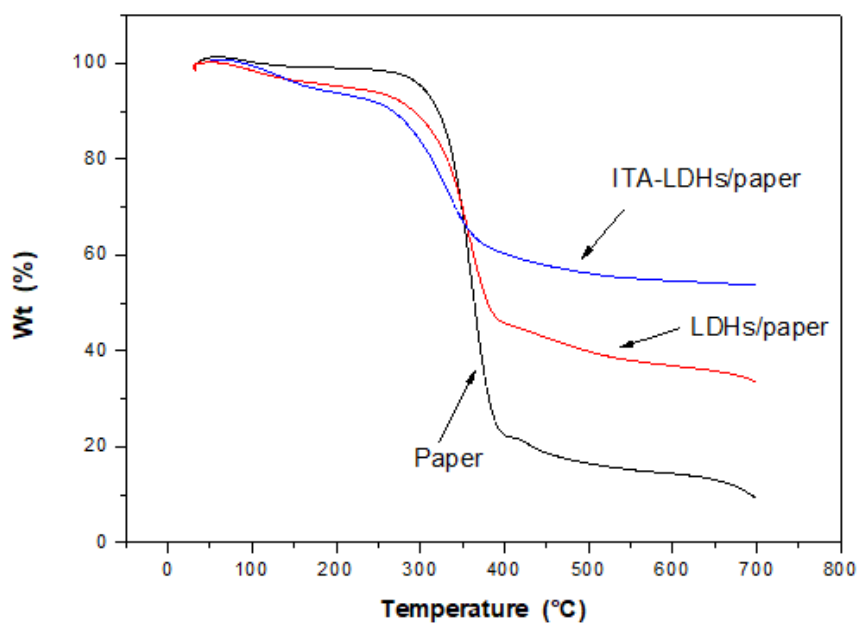

Fig. 7. Recorded TGA curves of paper handsheets made without LDHs, with unmodified LDHs, and with modified LDHs 


\section{Cone Calorimeter of Paper Handsheets with LDHs}

The total heat release rate (THR) and heat release rate (HRR) of flame retardant paper were measured by cone calorimeter, the addition of hydrotalcites were $20 \%$. From the data in Figs. 8 and 9, it can be seen that the paper without flame retardant burned rapidly after ignition, and the heat release rate increased rapidly, reaching a maximum of about 90 $\mathrm{s}$. The maximum heat release rate was $220.75 \mathrm{~kW} / \mathrm{m}^{2}$, and the time required for complete combustion was about $205 \mathrm{~s}$.

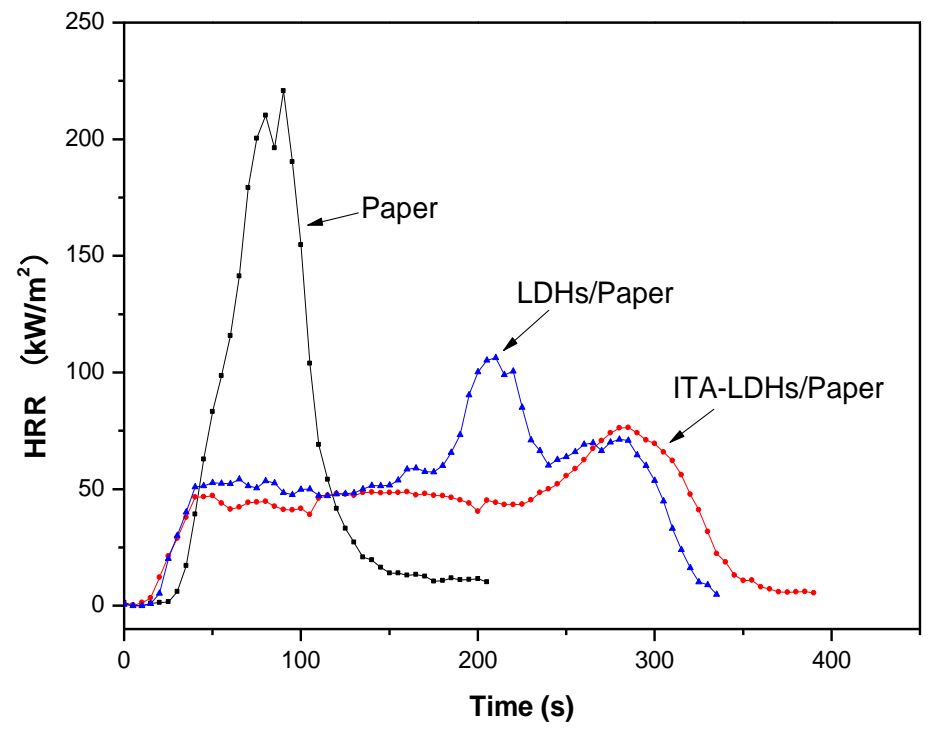

Fig. 8. HHR curves of paper handsheets made without LDHs, with unmodified LDHs, and with modified LDHs

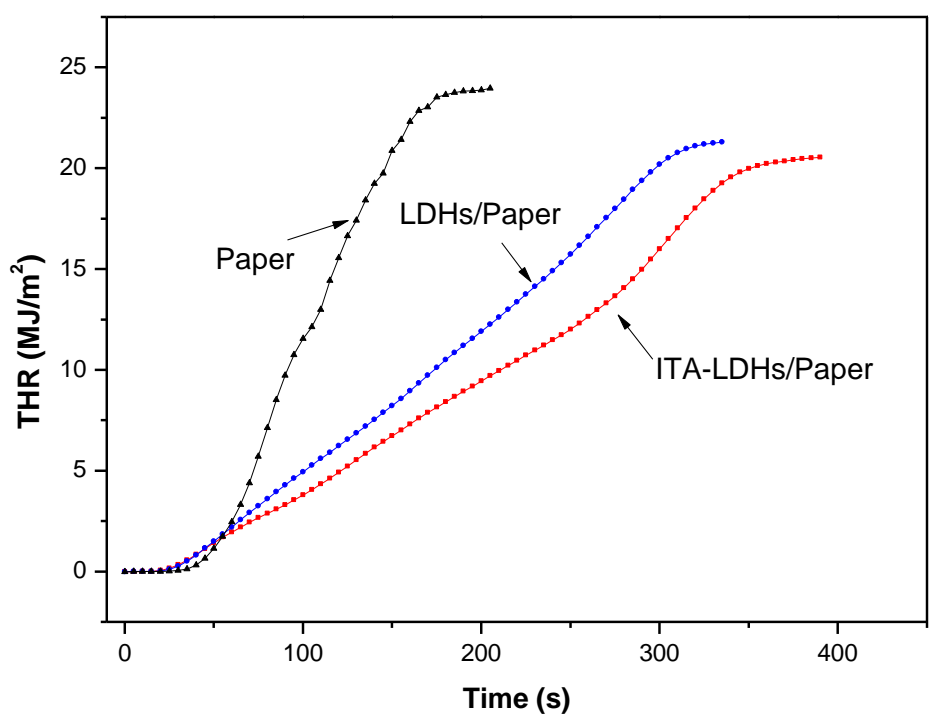

Fig. 9. THR curves of paper handsheets made without LDHs, with unmodified LDHs, and with modified LDHs

After ignition, the heat release rate of the flame retardant paper specimens with unmodified LDHs increased slowly. The time required for complete combustion was prolonged to $335 \mathrm{~s}$. The maximum heat release rate was $106.35 \mathrm{~kW} / \mathrm{m}^{2}$, and the total heat 
release decreased significantly. However, the heat release rate of flame retardant paper specimens with modified LDHs increased more slowly after ignition. The time required for complete combustion was extended to $390 \mathrm{~s}$, the maximum heat release rate was $76.1 \mathrm{~kW} / \mathrm{m}^{2}$, and the flame retardant effect was better.

\section{Analysis of Flame Retardant Mechanism}

The flame-retardant mechanism of the hydrotalcite was the solidification phase flame retardant mechanism. When the material was heated, the free water between the hydrotalcite layers was decomposed by heat and removed part of the heat. The heating was continued and the combined water between the hydrotalcite layers was removed and taken away from part of the heat source. Then, a part of the carbonate was decomposed into carbon dioxide from the heat, carbon dioxide is a non-flammable gas and when it escaped with water vapor it not only removed heat but also diluted the oxygen concentration in the air.

In the subsequent combustion process, the hydrotalcite was dehydrated, the interlayer hydrogen bonds disappeared, the laminate collapsed, and the lamellar structure dissociated between the materials. At the same time, itaconic acid and titanate coupling agents were decomposed by heat to form carbonate, pyrophosphate, and water vapor, providing a rich carbon source, promoting the formation of carbon into the matrix, forming a molten carbon layer. The collapsed hydrotalcite lamellar structure dissociated in the molten carbon layer to enhance the strength of the carbon layer coupled with the generation of water vapor, carbon dioxide, and other non-combustible gases. Such release made the molten carbon layer expand and removed part of the heat, and the expanded carbon layer gradually stabilized with continuous heating. The function of fire insulation and heat insulation protected the matrix so that the matrix did not continue to decompose to achieve the purpose of a flame retardant.

\section{Physical Strength Properties of Paper Handsheets Made with LDHs}

Figures 10 and 11 show that when no flame retardant was added, the tensile index and burst index of paper were $19.2 \mathrm{~N} \cdot \mathrm{m} \cdot \mathrm{g}^{-1}$ and $1.2 \mathrm{kPa} \cdot \mathrm{m}^{2} \cdot \mathrm{g}^{-1}$, respectively.

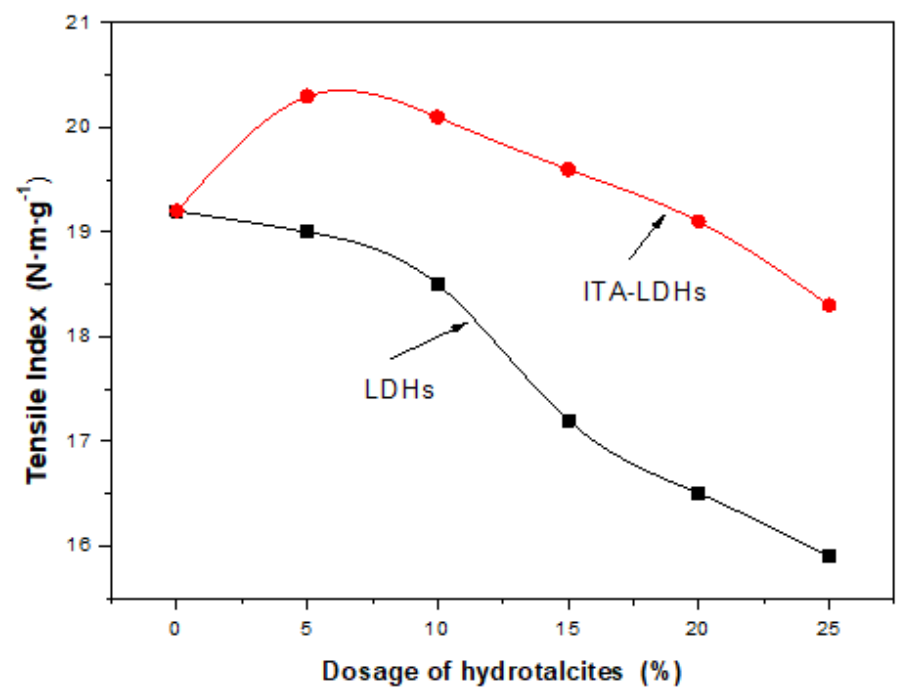

Fig.10. Effect of LDHs and ITA-LDHs dosage on the tensile index strength of paper handsheets 


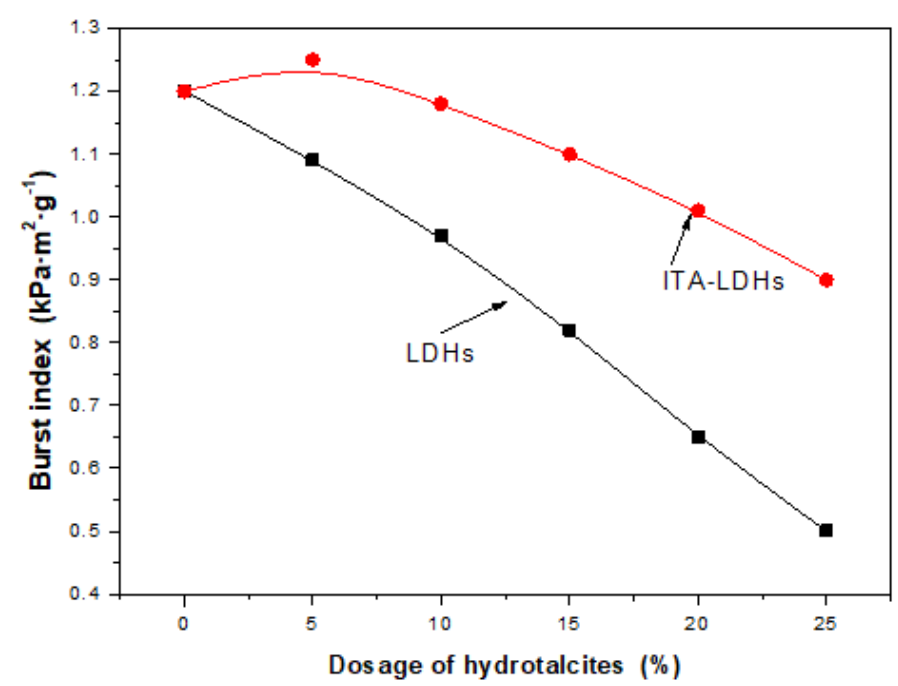

Fig. 11. Effect of LDHs and ITA-LDHs dosage on burst index strength of paper handsheets

The addition of LDHs (as flame retardants) during papermaking reduced the physical strength properties of the laboratory handsheets, especially with the unmodified LDHs. It was speculated that there was poor compatibility between the unmodified LDHs and the softwood fibers in the paper matrix, especially when the dosage of LDHs was more than $10 \%$ on pulp. In this case, the tensile and burst indices decreased with LDHs dosage increasing above $10 \%$ on pulp. The main reason for these reductions was that unmodified hydrotalcite affected the bonding force between the fibrils of the fibers and had an adverse effect on the strength index. Moreover, the interlayer spacing of the modified LDHs increased several times, the dispersion in fibers was improved, thereby improving the physical properties of the flame-retardant paper. Compared with the unmodified LDHs, the tensile index and burst index of the paper were noticeably improved.

As shown in Fig. 12, the whiteness of the paper handsheets increased as the dosage of LDHs or ITA-LDHs increased.

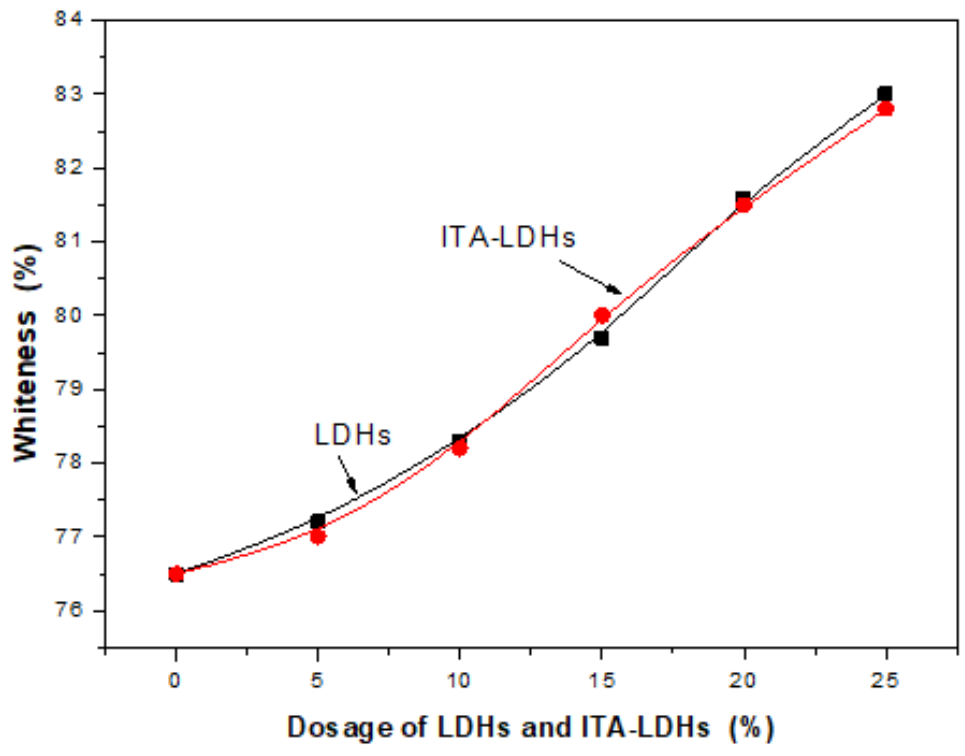

Fig. 12. Effect of LDHs and ITA-LDHs dosage on the whiteness of paper handsheets 
The whiteness of the handsheets increased from $c a .76 .5 \%$ to $83.0 \%$ when the dosage of either LDHs was $25 \%$ on pulp. Both hydrotalcites improved the whiteness of the paper as inorganic fillers at the same degree, because the light reflection coefficient of hydrotalcite was higher than that of fibers; moreover, as an inorganic filler, hydrotalcite can fill the gap between fibers and improved its reflective strength.

\section{CONCLUSIONS}

1. The organic modification of hydrotalcite with itaconic acid and titanate coupling agent NDZ-201 was successfully carried out and the interlayer spacing of the modified hydrotalcite was enlarged several times.

2. The flame retardancy of paper handsheets made with LDHs and ITA-LDHs fillers increased when compared to handsheets without the fillers. Handsheets made with ITA-LDHs fillers had better flame retardancy than handsheets made with unmodified LDHs. When the amount of filler addition was $20 \%$ on pulp, the LOI value of ITALDHs paper handsheets was $26.5 \%$, whereas this value was $24.1 \%$ for unmodified LDHs handsheets. The thermal stabilities of handsheets containing ITA-LDHs filler were higher than handsheets containing untreated LDHs, the HRR, and THR of were reduced.

3. The addition of unmodified hydrotalcites adversely affected the physical strength properties of the resulting paper handsheets. Modifying the LDH filler material (i.e., ITA-LDHs) improved its compatibility with softwood fibers within the formed paper matrix. This resulted in improved physical properties of the flame-retardant handsheets when compared to handsheets made with unmodified LDHs. The tensile and burst indices of the handsheets made with the modified LDHs were higher than those made with the unmodified LDHs. Both the unmodified and modified LDHs can be used as paper filler material to increase paper whiteness. When the dosage of LDHs was $25 \%$ on pulp, the whiteness of the formed handsheets was ca. $83 \%$ versus $76.5 \%$ for handsheets without LDHs.

\section{ACKNOWLEDGMENTS}

This research was supported by the Foundation of Key Laboratory of Pulp and Paper Science and Technology of the Ministry of Education (No. KF201706), and by the Shandong Province of China Key Research and Development Project (No. 201809120188). One of the authors expresses their deepest gratitude to Dr. Songlin Wang who provided valuable guidance during the course of conducting and reporting of this work.

\section{REFERENCES CITED}

Becker, C. M., Gabbardo, A. D., Wypych, F., and Amico, S. C. (2011). "Mechanical and flame-retardant properties of epoxy/Mg-Al LDH composites," Compos. Part AAppl. S. 42(2), 196-202. DOI: 10.1016/j.compositesa.2010.11.005

Choudary, B. M., Sateesh, M., Chowdari, N. S., Kantam, M. L., and Bojja, S. (2002). 
"Layered double hydroxide supported nanopalladium catalyst for Heck-, Suzuki-, Sonogashira-, and Stille-type coupling reactions of chloroarenes," J. Am. Chem. Soc. 124(15), 14127-14136. DOI: 10.1021/ja026975w

Costantino, U., Nocchetti, M., Sisani, M., and Vivani, R. (2009). "Recent progress in the synthesis and application of organically modified hydrotalcites," Int. J. Struct. Phys. Chem. Aspects Crystal. Mater. 224(5-6), 273-281. DOI:

10.1524/zkri.2009.1153

Gardolinski, J. E., Carrera, L. C. M., Cantão, M. P., and Wypych, F. (2000). "Layered polymer-kaolinite nanocomposites," J. Mater. Sci. 35(12), 3113-3119. DOI: 10.1023/a:1004820003253

GB/T 12914 (2008). "Paper and board - Determination of tensile properties," Standardization Administration of China, Beijing, China.

GB/T 14656 (2009). "Test method for burning behavior of flame-retardant paper and board," Standardization Administration of China, Beijing, China.

GB/T 454 (2002). "Paper - Determination of bursting strength," Standardization Administration of China, Beijing, China.

Horrocks, A. R. (1996). "Developments in flame retardants for heat and fire resistant textiles- the role of char formation and intumescence," Polym. Degrad. Stabil. 54(23), 143-154. DOI: 10.1016/S0141-3910(96)00038-9

Horrocks, A. R., Wang, M. Y., Hall, M. E., Sunmonu, F., and Pearson, J. S. (2015). "Flame retardant textile back coatings. Part 2. Effectiveness of phosphoruscontaining flame retardants in textile back-coating formulations," Polym. Int. 49(10), 1079-1091. DOI: 10.1002/1097-0126(200010)49:10<1079::AID-PI468>3.0.CO;2-Z

Kalali, E. N., Wang, X., and Wang, D. Y. (2015). "Functionalized layered double hydroxide-based epoxy nanocomposites with improved flame retardancy and mechanical properties," J. Mater. Chem. A 3(13), 6819-6826. DOI: $10.1039 / \mathrm{c} 5 \mathrm{ta0} 0010 \mathrm{f}$

Khan, A. I., and O'Hare, D. (2002). "Intercalation chemistry of layered double hydroxides: Recent developments and applications," ChemInform 34(20), 31913198. DOI: 10.1002/chin.200320250

Köklükaya, O., Carosio, F., Grunlan, J. C., and Wågberg, L. (2015). "Flame-retardant paper from wood fibers functionalized via layer-by-layer assembly," ACS Appl. Mater. Inter. 7(42), 23750-23759. DOI: 10.1021/acsami.5b08105

Li, Y., Zou, J., Zhou, S., and Chen, Y. (2014). "Effect of expandable graphite particle size on the flame retardant, mechanical, and thermal properties of water-blown semirigid polyurethane foam," Journal of Applied Polymer Science 131(3), 3338. DOI:10.1002/app.39885

Li, F., and Duan, X. (2006). “Applications of layered double hydroxides,” in: Layered Double Hydroxides. Structure and Bonding, Vol. 119, X. Duan and D. G. Evans (eds.), Springer-Verlag, Berlin, Germany, pp. 193-223. DOI: 10.1007/430_007

Liu, Z., Ma, R., Osada, M., Iyi, N., Ebina, Y., and Takada, K. (2006). "Synthesis, anion exchange, and delamination of Co-Al layered double hydroxide: Assembly of the exfoliated nanosheet/polyanion composite films and magneto-optical studies," $J$. Am. Chem. Soc. 128(14), 4872-4880. DOI: 10.1021/ja0584471

Mahmoud, R., Moaty, S. A., Mohamed, F., and Farghali, A. (2017). "Comparative study of single and multiple pollutants system using Ti-Fe chitosan LDH adsorbent with high performance in wastewater treatment," J. Chem. Eng. Data 62(11), 48724880. DOI: $10.1021 /$ acs.jced.7b00453 
Nassar, M. M., Fadali, O. A., Khattab, M. A., and Ashour, E. A. (2015). "Thermal studies on paper treated with flame-retardant," Fire Mater. 23(3), 125-129. DOI: 10.1002/(SICI)1099-1018(199905/06)23:3<125::AID-FAM677>3.0.CO;2-

Pereira, C. M. C., Herrero, M., Labajos, F. M., Marques, A. T., and Rives, V. (2009). "Preparation and properties of new flame retardant unsaturated polyester nanocomposites based on layered double hydroxides," Polym. Degrad. Stabil. 94(6), 939-946. DOI: 10.1016/j.polymdegradstab.2009.03.009

QB/T 2804 (2006). "Paper and board - Measurement of brightness (45/0)," Standardization Administration of China, Beijing, China.

Reichle, W. T. (1986). "Synthesis of anionic clay minerals (mixed metal hydroxides, hydrotalcite)," Solid State Ionics 22(1), 135-141. DOI:

Shi, Y., Chen, F., Yang, J., and Zhong, M. (2010). "Crystallinity and thermal stability of LDH/polypropylene nanocomposites," Appl. Clay Sci. 50(1), 87-91. DOI: 10.1016/j.clay.2010.07.007

Si, Y., and Guo, Z. (2016). "Eco-friendly functionalized superhydrophobic recycled paper with enhanced flame-retardancy," J. Colloid Interf. Sci. 477, 74-82. DOI: 10.1016/j.jcis.2016.05.044

Standardization Administration of China, Beijing, China.

Wang, Q., and O'Hare, D. (2012). "Recent advances in the synthesis and application of layered double, hydroxide (LDH) nanosheets," Chem. Rev. 112(7), 4124-4155. DOI: $10.1021 / \mathrm{cr} 200434 \mathrm{v}$

Wang, S. L., and Song, X. M. (2010). "Synthesize and application of Mg-Al hydrotalcite in flame retardant paper preparation," Adv. Mat. Res. 174, 362-365. DOI: $10.4028 / w w w . s c i e n t i f i c . n e t / A M R .174 .362$

Weinstock, I. A., Atalla, R. H., Reiner, R. S., Moen, M. A., Hammel, K. E., Houtman, C. J., Hill, C. L., and Harrup, M. K. (1997). "A new environmentally benign technology for transforming wood pulp into paper. Engineering polyoxometalates as catalysts for multiple processes," J. Mol. Catal. A-Chem. 116(1-2), 59-84. DOI: 10.1016/S13811169(96)00074-X

Yan, Y., Gu, X., Li, L., Li, H., Sun, J., and Zhang, S. (2017). "Preparation and characterization of intumescent flame retardant biodegradable poly(lactic acid) nanocomposites based on sulfamic acid intercalated layered double hydroxides," Fibers and Polymers 18(11), 2060-2069.DOI:10.1007/s12221-017-1153-2

Zhang, L., Liang, S., Zhang, L., and Chen, Z. (2016). "Properties of fire-retardant medium density fiberboard made with inorganic magnesium-aluminum layered double hydroxide (MgAl-LDH)," China Wood Industry 30(6), 9-13.

DOI: $10.19455 /$ j.mcgy.20160602.

Article submitted: November 28, 2018; Peer review completed: February 24, 2019; Revised version received and accepted: March 24, 2019; Published: March 29, 2019. DOI: 10.15376/biores.14.2.3991-4005 